\title{
Accuracy of micro four-point probe measurements on inhomogeneous samples: A probe spacing dependence study
}

Wang, Fei; Petersen, Dirch Hjorth; Østerberg, Frederik Westergaard; Hansen, Ole

Published in:

17th International Conference on Advanced Thermal Processing of Semiconductors, 2009. RTP '09

Link to article, DOI:

10.1109/RTP.2009.5373449

Publication date:

2009

Document Version

Publisher's PDF, also known as Version of record

Link back to DTU Orbit

Citation (APA):

Wang, F., Petersen, D. H., Østerberg, F. W., \& Hansen, O. (2009). Accuracy of micro four-point probe measurements on inhomogeneous samples: A probe spacing dependence study. In 17th International Conference on Advanced Thermal Processing of Semiconductors, 2009. RTP '09 (pp. 1-6). IEEE. https://doi.org/10.1109/RTP.2009.5373449

\section{General rights}

Copyright and moral rights for the publications made accessible in the public portal are retained by the authors and/or other copyright owners and it is a condition of accessing publications that users recognise and abide by the legal requirements associated with these rights.

- Users may download and print one copy of any publication from the public portal for the purpose of private study or research.

- You may not further distribute the material or use it for any profit-making activity or commercial gain

- You may freely distribute the URL identifying the publication in the public portal 
$17^{\text {th }}$ IEEE International Conference on Advanced Thermal Processing of Semiconductors - RTP 2009

\title{
Accuracy of Micro Four-Point Probe Measurements on Inhomogeneous Samples: A Probe Spacing Dependence Study
}

\author{
Fei Wang ${ }^{1}$, Dirch H. Petersen ${ }^{1,2}$, Frederik W. Osterberg ${ }^{1}$, Ole Hansen ${ }^{1,3}$ \\ ${ }^{1}$ Department of Micro- and Nanotechnology, Technical University of Denmark, \\ DTU Nanotech Building 345 East DK-2800 Kgs. Lyngby, Denmark and \\ ${ }^{2}$ Capres A/S, Scion-DTU, Building 373, DK-2800 Kgs. Lyngby, Denmark and \\ ${ }^{3}$ Danish National Research Foundation's Center for Individual Nanoparticle Functionality (CINF), \\ Technical University of Denmark, DK-2800 Kgs. Lyngby, Denmark \\ Email: Fei.Wang@nanotech.dtu.dk
}

\begin{abstract}
In this paper, we discuss a probe spacing dependence study in order to estimate the accuracy of micro four-point probe measurements on inhomogeneous samples. Based on sensitivity calculations, both sheet resistance and Hall Effect measurements are studied for samples (e.g. laser annealed samples) with periodic variations of sheet resistance, sheet carrier density, and carrier mobility. With a variation wavelength of $\lambda$, probe spacings from $0.001 \lambda$ to $100 \lambda$ have been applied to characterize the local variations. The calculations show that the measurement error is highly dependent on the probe spacing. When the probe spacing is smaller than 1/40 of the variation wavelength, micro four-point probes can provide an accurate record of local properties with less than $1 \%$ measurement error. All the calculations agree well with previous experimental results.
\end{abstract}

\section{INTRODUCTION}

Along with the continuous downscaling of the critical dimension in semiconductor processes, sheet materials such as ultra shallow junctions (USJ) are widely used in the process development. Characterization and monitoring of implant and annealing technologies for USJ is a significant metrology challenge. For several decades, conventional four-point probe measurement has been a useful metrology technique to characterize sheet resistance, sheet carrier density, and carrier mobility $[1,2]$ when combined with Hall Effect measurements $[3,4]$. However, conventional four-point probes can cause large measurement errors on advanced micro or even nano-scale structures like USJ, unless the probe spacing can also be down scaled simultaneously with the devices under test [5]. Recently, in-line micro four-point probe (M4PP) measurements have been proven to be an accurate method for characterization of USJ sheet resistance [6,7]. The accuracy of the measurements has been studied for small samples with dimensions comparable to the probe spacing [8]. Moreover, micro Hall effect measurements with M4PP have also been applied, and carrier mobility, sheet carrier density and sheet resistance of USJ have been accurately measured e.g. on cleaved non-patterned wafers $[9,10,11]$.

The measurement results are actually mean values of the local properties across the measured sample which is perfect

978-1-4244-3815-0/09/\$25.00 CIEEE with ideally homogeneous samples. Real samples such as laser annealed junctions, however, may not be perfectly homogeneous and can exhibit local variations in electrical properties related to e.g. the stitching overlays and inhomogeneity of the laser beam (e.g. a diode array) used for annealing. Recently, we have calculated the sensitivities of micro four-point probe sheet resistance and Hall Effect measurements to small variations in the local properties [12].

In this study, the sensitivity values are used to estimate the measured variation according to periodic variations on inhomogeneous samples. Furthermore, the dependence of the measurement error on the probe spacing is studied in detail.

\section{METHOD}

For an inhomogeneous sample, with a small change of the local sheet resistance, $R_{L}$, the local sheet carrier density, $N_{L}$, or the local carrier mobility, $\mu_{L}$, in a small area, $\Delta A$, the measured values of the sheet resistance, $R_{S}$, and the Hall sheet resistance, $R_{h}$, will also change as a result. Therefore, the sensitivity of the measured values $T\left(T=R_{S}, R_{h}, \mu\right.$ or $\left.N_{S}\right)$ to the local properties $t\left(t=R_{L}, N_{L}\right.$ or $\left.\mu_{L}\right)$, can be defined as a dimensionless sensitivity variable $S_{t}^{T}$ :

$$
S_{t}^{T}=\lim _{\Delta t, \Delta A \rightarrow 0} p^{2} \frac{\Delta T / T}{\Delta t / t} \frac{1}{\Delta A}=p^{2} \frac{\partial^{2} T}{\partial t \partial A} \frac{t}{T}
$$

where $p$ is the probe spacing; $\Delta t$ is the local variation and $\Delta T$ is the measured variation; $\mu$ and $N_{S}$ are the measured mobility and sheet carrier density, respectively. Two-dimensional (2D) sensitivity values of the sheet resistance and the Hall Effect measurements have been plotted in [12] and will be used in this study.

Based on the sensitivity definition a small 2D variation of the local property $\Delta t(x, y)$ across an inhomogeneous sample will result in a measured variation of $\Delta T$ as follows:

$$
\frac{\Delta T}{T_{0}}=\iint_{\Omega} \frac{\Delta t(x, y)}{t_{0}} \cdot S_{t}^{T} / p^{2} d \Omega
$$

where $t_{0}$, and $T_{0}$ are the mean values corresponding to an 


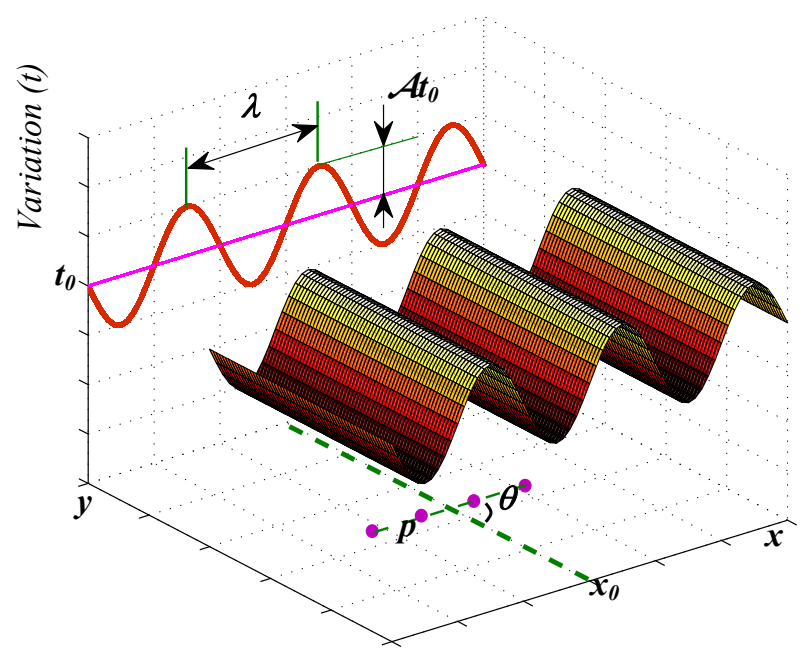

Fig. 1 Schematic view of the local property variation and the micro four-point probes for scanning measurement.

equivalent homogeneous sample. Variations in the most sensitive area near the probes can affect the measurement results significantly while regions further away only contribute slightly.

In this study, we will study an inhomogeneous sample with a small periodic parameter variation defined by (3):

$$
\frac{\Delta t(x, y)}{t_{0}} \equiv \mathcal{A} \sin (2 \pi x / \lambda)
$$

As schematically shown in Fig. 1, the local property $t$ has a sinusoidal variation in the $x$ direction with a scaled amplitude of $\mathcal{A}$ and wavelength of $\lambda$, while $t$ does not vary in the $y$ direction. The probes are always assumed to be scanned across the sample in the $x$ direction to trace the local variation. As for the line of the probe, the four probes can be placed in any direction for sheet resistance measurement on an infinite film, thus forming an angle $\theta$ between the line of the probe and the $y$ direction. For the Hall Effect measurement on a cleaved wafer, the wafer is assumed to be cleaved along the $x$ direction, and since the line of the probe must be parallel to the cleaved boundary, the angle $\theta$ is 90 degrees in this case.

Using equations (2) and (3) the measured variation can be expressed as:

$$
\frac{\Delta T}{T_{0}}=\int_{\Omega} \mathcal{A} \sin (2 \pi x / \lambda) \cdot S_{t}^{T} / p^{2} d \Omega
$$

When the four point probes are moved along the $x$ direction to scan the local variation, the measured variation at $x=x_{0}$ becomes:

$$
\begin{aligned}
\frac{\Delta T\left(x_{0}\right)}{T_{0}} & =\int_{\Omega} \mathcal{A} \sin (2 \pi x / \lambda) \cdot S\left(x-x_{0}, y\right) / p^{2} d \Omega \\
& =\int_{x} \mathcal{A} \sin (2 \pi x / \lambda) \cdot S_{l}\left(x-x_{0}\right) / p d x
\end{aligned}
$$

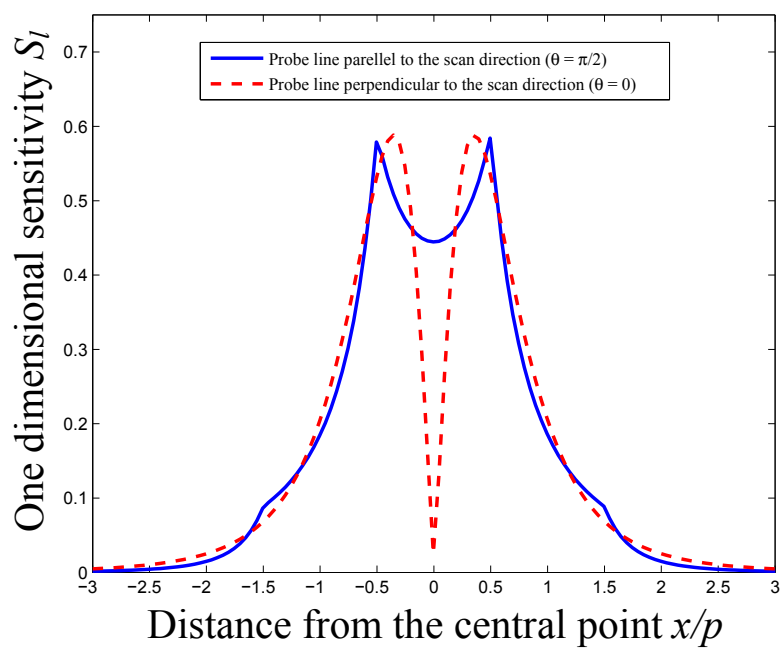

Fig. 2 1D sensitivity of the measured sheet resistance to the local sheet resistance for infinite sample.

$$
=\int_{x} \mathcal{A} \sin \left[2 \pi\left(x+x_{0}\right) / \lambda\right] \cdot S_{l}(x) / p d x
$$

since the local property is constant in the $y$ direction. In Equation 5, $S_{l}$ is the one-dimensional (1D) sensitivity, which is defined as the line integral of the $2 \mathrm{D}$ sensitivity in the $y$ direction divided by the probe pitch:

$$
S_{l}(x)=\int_{l} S(x, y) / p d y
$$

From equation (5), it follows that also $\mathcal{A}$ is periodic with the wavelength of $\lambda$,

$$
\frac{\Delta T}{T_{0}} \equiv \mathcal{A}_{m} \sin \left(2 \pi x / \lambda+\varphi_{s}\right)
$$

with the scaled amplitude $\mathcal{A}_{m}$ of the measurement result and the phase shift $\varphi_{s}$ relative to the local variation.

The sensitivity values used here were simulated with dual configuration sheet resistance measurements, while for the Hall Effect measurements the dual point three configuration method was used, just as in the practical measurements [11, 12].

\section{RESULTS AND DISCUSSION}

\section{Sheet Resistance Measurement}

First, we calculate the 1D sensitivity of measured sheet resistance to the local sheet resistance, according to the different probe line directions. Since the line of the probe could have any angle with the scanning direction for an infinite film, we shall study two typical cases: the probe line perpendicular to the scanning direction $(\theta=0)$, and the line of the probe parallel to the scanning direction $\left(\theta=90^{\circ}\right)$. The 1D sensitivities are plotted as a function the normalized distance $x / p$ from the probe centre. As shown in Fig. 2, the 1D 


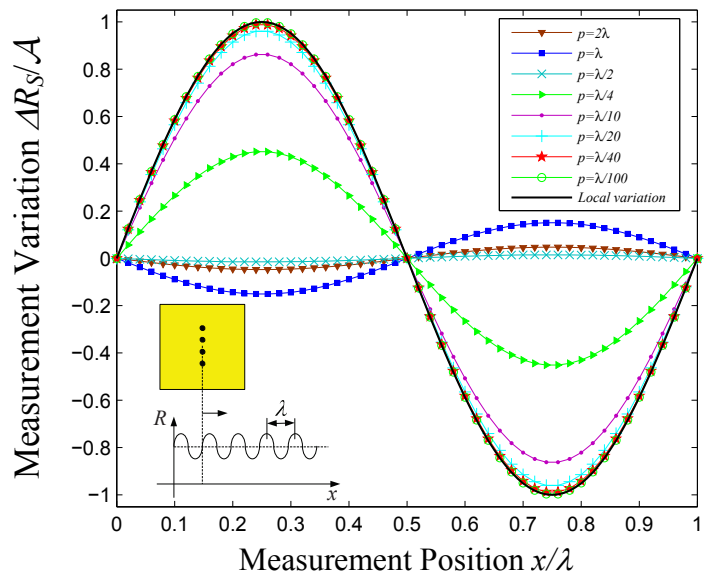

(a)

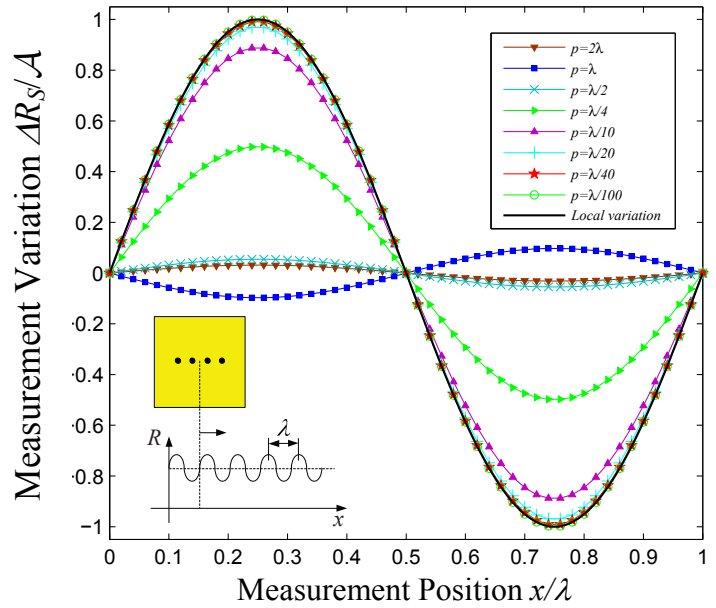

Fig. 3 Sheet resistance measurement result with different probe spacing according to a sinusoidal variation of local sheet resistance: (a) Probe line perpendicular to the scanning direction; (b) Probe line parallel to the scanning direction

sensitivities show two peak values around $0.5 p$ from the probe centre for both probe orientations. For the probe line parallel to the scanning direction, the sensitivity is always higher than 0.1 within a distance of $1 p$ from the probe centre, while for the probe line perpendicular to the scanning direction, the sensitivity is almost zero at the probe centre. Thus the measured sheet resistance is not sensitive to the local sheet resistance on the probe line (except the probe points).

This canyon like distribution of sensitivity makes the measurement in the perpendicular situation less concentrated than the parallel one, which will cause a slightly larger measurement error at identical probe spacing.

Fig. 3 shows the simulated measurement result in a scan across one full wavelength of the local sheet resistance variation using eight different probe spacings. For both directions, the smaller the probe spacing used, the closer the measured result is to the local variation. About $99 \%$ of the local variation can be resolved when the probe spacing is smaller than $\lambda / 40$, while less than $40 \%$ can be detected if the probe spacing is larger than $\lambda / 4$. Furthermore, when a probe

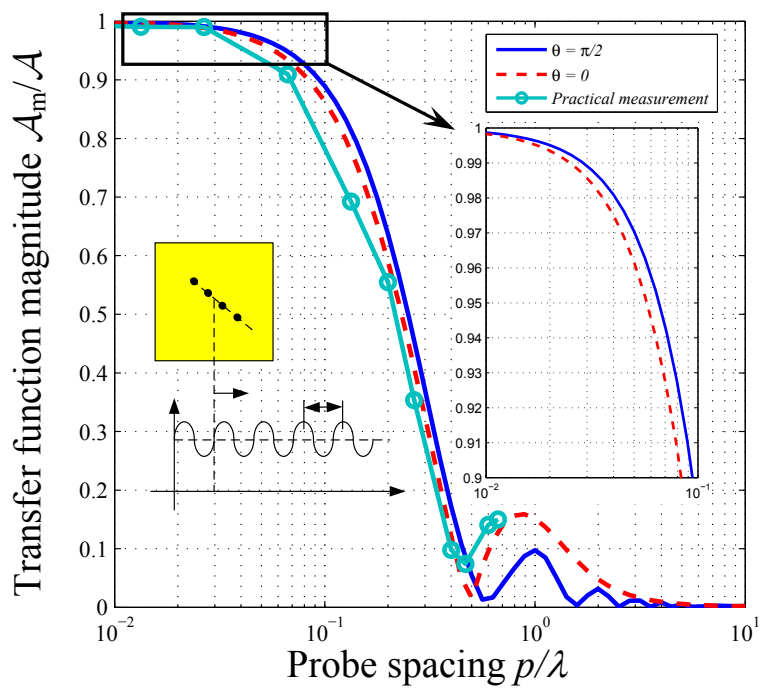

Fig. 4 The sheet resistance measurement transfer function as a function of the probe spacing $\mathrm{p}$ for a local sheet resistance variation with a wavelength of $\lambda$. For comparison, the previously reported measurements are included [5].

spacing approximately equal to the variation wavelength is applied, a reversal phase shift is observed because the most sensitive area is just half of the variation wavelength away from the measurement point. As discussed above, the measurement variation in Fig. 3(a) $(\theta=0)$ is always slightly smaller than that in Fig. 3(b) $\left(\theta=90^{\circ}\right)$ when the same probe spacing is used. This is more clearly demonstrated in Fig. 4 where accuracy of the sheet resistance measurement is shown as a spatial frequency transfer function of the probe spacing. It can be seen that the measurement accuracy decreases remarkably with an increase of the probe spacing. If a measurement error of less than $1 \%$ is desired (which means a total measurement error of $0.1 \%$ for local variation amplitude of $10 \%$, as often in the semiconductor industry), a small probe spacing of $\lambda / 40$ is indispensable. This is approximately $20 \mu \mathrm{m}$ probe pitch for a typical laser inhomogeneity wavelength of $0.75 \mathrm{~mm}$. For comparison, Fig. 4 includes the measured variation in previously reported practical measurements [5], which agrees well with our calculations.

\section{Hall Effect Measurement}

Unlike the sheet resistance measurement, the Hall Effect measurement is done on a half-plane film such as a cleaved wafer using the dual point three configurations technique [11]. The probe line is parallel to the cleaved boundary which is aligned to the $x$ direction and therefore, $\theta$ is always 90 degrees.

The measured properties studied in [12] are the sheet resistance and Hall sheet resistance, while the local variations are the local sheet carrier density and local carrier mobility. Using the sensitivity values, we can first calculate the sensitivities of the measured sheet resistance and Hall sheet resistance to local sheet carrier density $\left(N_{L}\right)$ and local carrier mobility $\left(\mu_{L}\right)$, respectively. The four resulting 1D sensitivities 


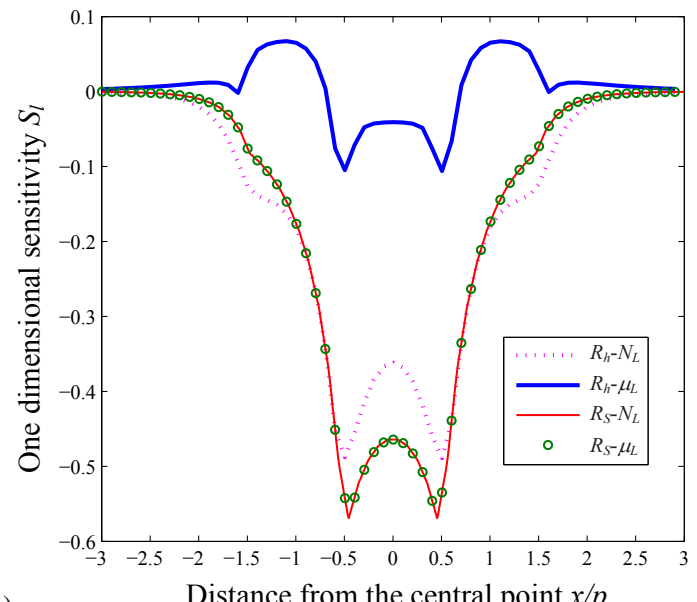

(a)

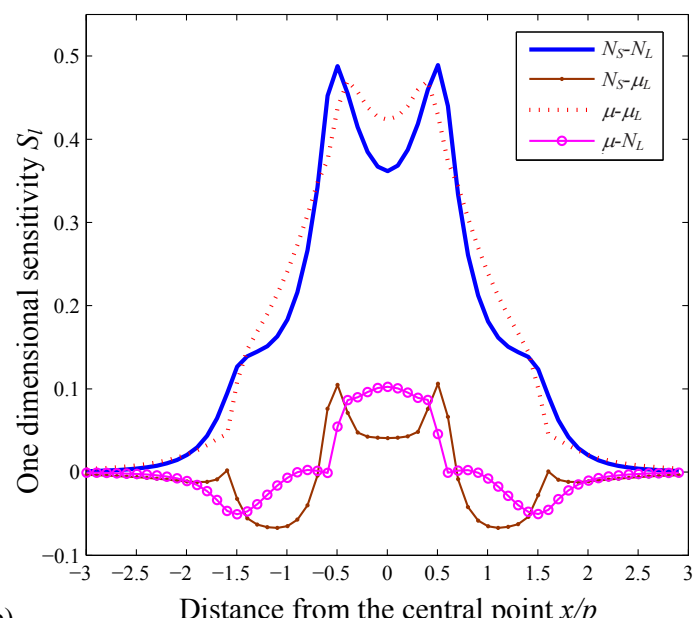

(b)

Fig. 5 1D sensitivities of (a) the sheet resistance and the Hall sheet resistance; (b) the sheet carrier density and the carrier mobility to variations of the local sheet carrier density and the local carrier mobility for Hall effect measurements on a cleaved wafer with a single boundary.

are shown in Fig. 5(a). The measured sheet resistance has almost the same sensitivity to the local sheet carrier density and the local mobility, which makes the two 1D sensitivity plots overlap each other. The two plots show similar shape as the sensitivity of local sheet resistance shown in the Fig. 2 as expected.

Using the known sensitivities of sheet resistance and Hall sheet resistance, the sensitivities of the extracted sheet carrier density and carrier mobility can also be calculated:

$$
S_{t}^{N_{S}}=-S_{t}^{R_{H}} \text { and } S_{t}^{\mu}=S_{t}^{R_{H}}-S_{t}^{R_{S}}
$$

Fig. 5(b) shows the calculated 1D sensitivity of $N_{S}$ and $\mu$ to the local variations in sheet carrier density and mobility, respectively. The sensitivities of $N_{S}-N_{L}$ and $\mu-\mu_{L}$ has a similar shape as that of $R_{S}-R_{L}$ shown in Fig. 2. The sensitivities in Fig. 5 (b) are further used to calculate the measurement accuracy for different probe spacing. The extracted sheet carrier density and mobility are shown in Fig. 6(a) and Fig. 6(b), respectively, as a function of measurement position across a full

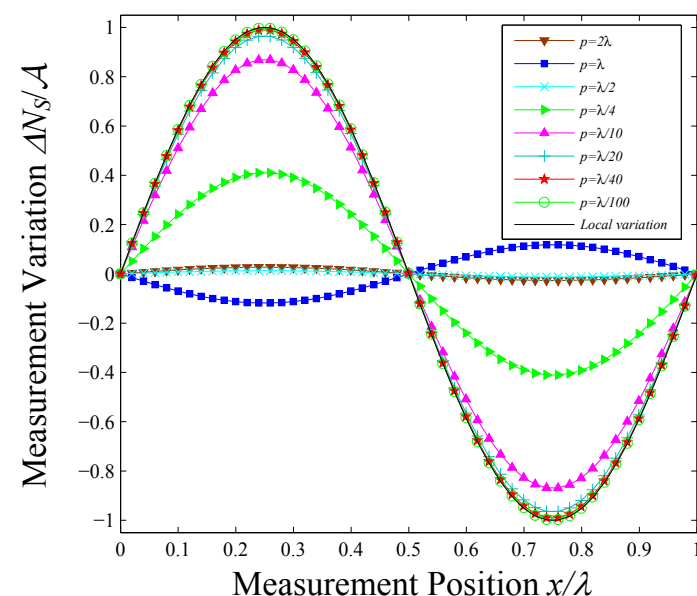

(a)

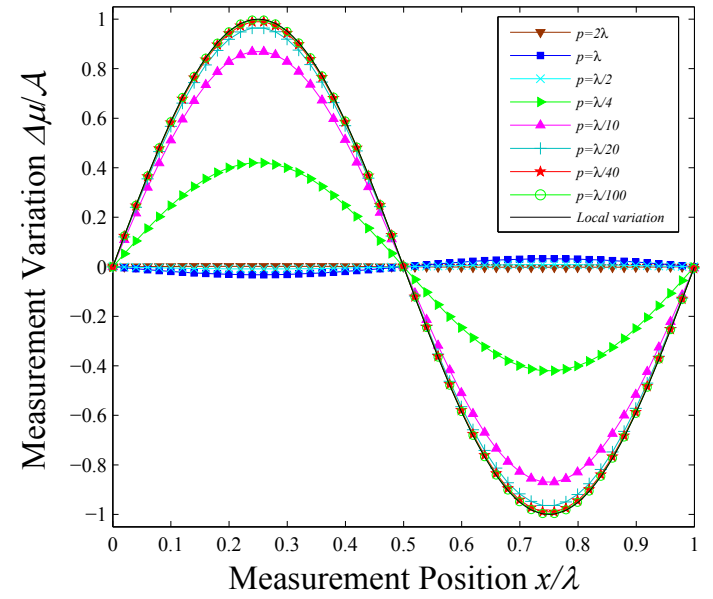

Fig. 6 (a) Variation of the measured sheet carrier density in Hall effect measurement to a sinusoidal variation of the local sheet carrier density; (b) Variation of the measured carrier mobility in Hall effect measurement to a sinusoidal variation of the local carrier mobility.

wavelength of sheet carrier density and mobility variation. Local variations can be accurately traced when the probe spacing is as small as $1 / 40$ of the variation wavelength.

Fig. 5(b) also shows the relatively small cross sensitivity values $N_{S}-\mu_{L}$ and $\mu-N_{L}$ for extracted mobility to sheet carrier density and extracted sheet carrier density to mobility, respectively. The cross sensitivities are mainly caused by the position error suppression calculation when two measurement points are used $[11,12]$. As a result, the measurements for the sheet carrier density and the carrier mobility are not totally independent of each other. Fig. 7 for instance, shows a variation of the measured carrier mobility when a sinusoidal variation of the local sheet carrier density is assumed, even though the local carrier mobility is constant across the sample. The scaled amplitude of the local variation of $N_{S}$ is set to $10 \%$ and this results in a maximum variation of $1.2 \%$ for the measured mobility when the probe spacing is $1 / 4$ of the variation wavelength while at other probe spacings the cross sensitivity is smaller. The cross sensitivity is a source of measurement error which should be controlled well during a 


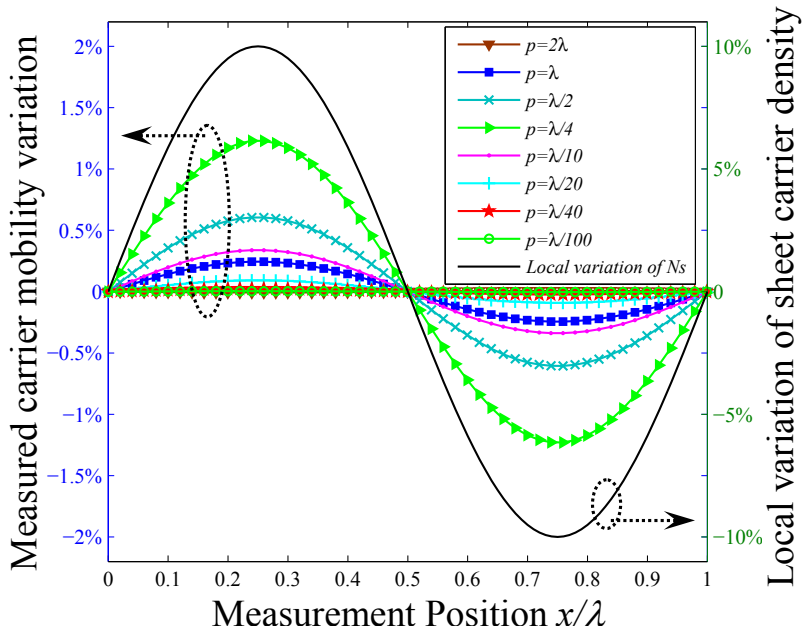

Fig. 7 Variation of the measured carrier mobility to a sinusoidal variation of the local sheet carrier density.

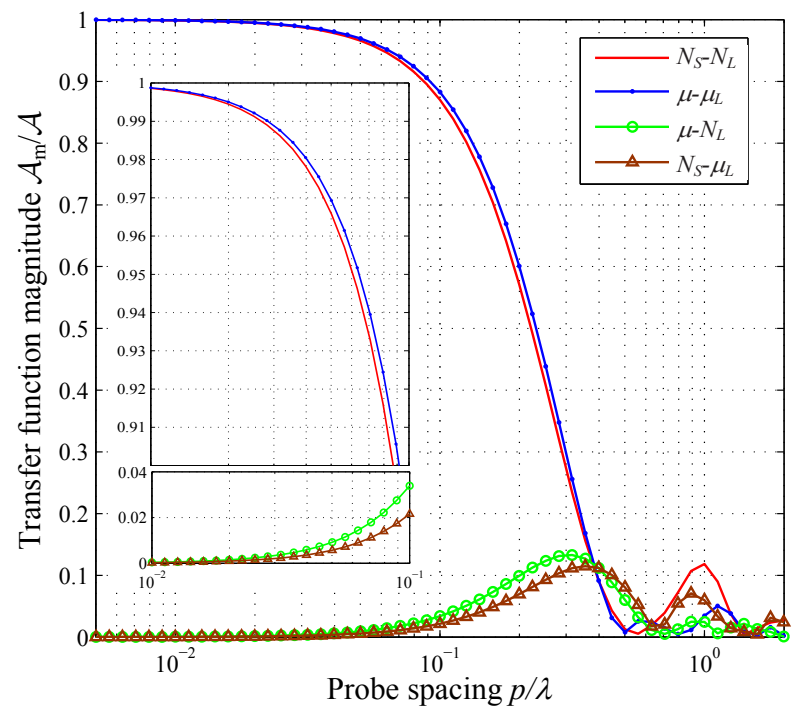

Fig. 8 The accuracy of Hall effect measurements as a function of the probe spacing $p$ for a local sheet resistance variation with a wavelength of $\lambda$.

\section{practical measurement.}

Fig. 8 shows the four spatial frequency transfer functions of measured sheet carrier density and mobility from sample sheet carrier density and mobility, respectively, as a function of the probe spacing for the micro Hall Effect measurements. It follows that $99 \%$ of the local variation is resolved when the probe spacing is smaller than $\lambda / 40$, while at the same time the cross sensitivity is reduced to less than $1 \%$.

\section{Measurement with square variation}

The calculation method is also applicable for a square wave parameter variation. The square wave may easily be analyzed using a Fourier series. Here, however, we calculated the measured result directly using the $1 \mathrm{D}$ sensitivity and the square wave variation. For instance, a local square wave sheet resistance variation with the wavelength of $\lambda$ is assumed on an

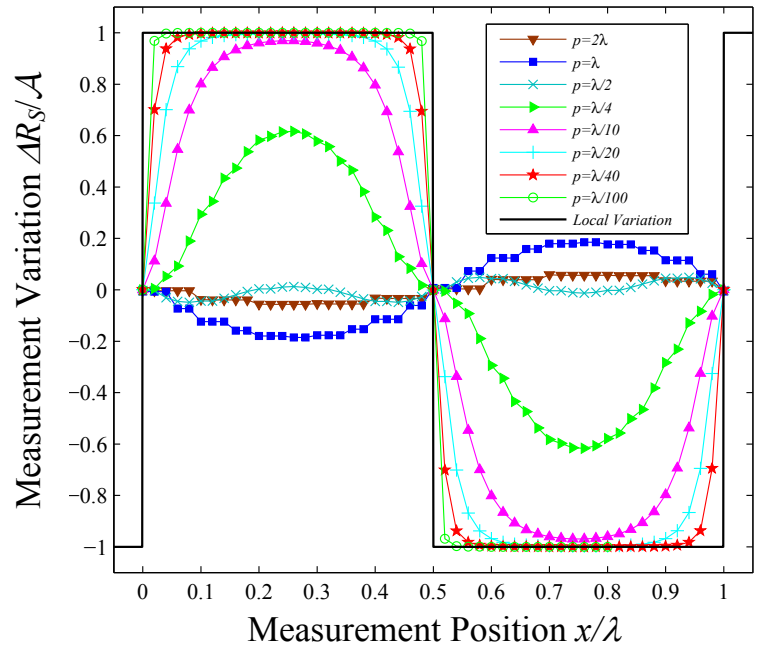

Fig. 9 The measured variations of the sheet resistance with different probe spacing, according to a local sheet resistance variation of square wave with a wavelength of $\lambda$.

infinite sample. The measured sheet resistance variations shown in Fig. 9 were calculated with eight different probe spacings. When the probe spacing is larger than $\lambda / 4$, the measured variation is similar to a sinusoidal wave. When the probe spacing is smaller than $0.1 \lambda$, the measured variation becomes a square wave with a rounded corner which represents the ambiguous response to the shifting boundary. When the probe spacing is as small as $0.01 \lambda$, the square wave variation is well reproduced.

\section{CONCLUSION}

We have studied the accuracy of M4PP measurement on inhomogeneous samples as a function of the probe spacing using calculations based on previously reported sensitivities. For both sheet resistance and micro Hall Effect measurements, the measured results are calculated at different probe spacings. We have proved that the measurement accuracy decreases significantly with increasing probe spacing. For local sinusoidal variation with wavelength of $\lambda$, the probe spacing must be less than $\lambda / 40$ when $1 \%$ measurement error is desired. The cross sensitivity from mobility to sheet carrier density and from sheet carrier density to mobility is also well controlled with small probe spacing for Hall Effect measurements. The calculations prove that the measured sheet resistance, sheet carrier density and carrier mobility accurately reflect the sinusoidal variations when the probe spacing is smaller than $\lambda / 40$. For the square wave variation, however, the absolute measurement accuracy is a function of the chosen probe spacing, the spatial frequency transfer function and the spatial Fourier frequencies.

\section{ACKNOWLEDGMENT}

We are grateful for the financial support from Copenhagen Graduate School for Nanoscience and Nanotechnology 
(C:O:N:T), the Danish Research Agency (FTP), and Danish National Advanced Technology Foundation. Center for Individual Nanoparticle Functionality (CINF) is sponsored by The Danish National Research Foundation. We would also like to thank Erik Rosseel and Wilfried Vandervorst from IMEC for their continuous support and good discussions.

\section{REFERENCES}

[1]. L. B. Valdes, "Resistivity measurements on germanium for transistors," Proc. IRE, 42, pp. 420-427, 1954

[2]. F. M. Smits, "Measurement of sheet resistance with the four point probe," Bell Syst. Tech. J. 37, pp. 711-718, 1958.

[3]. E.H. Hall, "On a new action of the magnet on electric currents," Am. J. Math., 2, pp. 287-292, 1879.

[4]. L. J. van der Pauw, "A method of measuring the resistivity and Hall coefficient on Lamellae of arbitrary shape," Philips Res. Rep., vol. 13, p. 1, 1958.

[5]. D. H. Petersen, R. Lin, T. M. Hansen, E. Rosseel, W. Vandervorst, C. Markvardsen, D. Kjær, and P. F. Nielsen, "Comparative study of size dependent four-point probe sheet resistance measurement on laser annealed ultra-shallow junctions," J. Vac. Sci. Technol. B 26, pp. 362-367, 2008.
[6]. T. Clarysse, A. Moussa, F. Leys, R. Loo, W. Vandervorst, M. C. Benjamin, R. J. Hillard, V. N. Faifer, M. I. Current, R. Lin, and D. H. Petersen, "Accurate sheet resistance measurement on ultra-shallow profiles," Mater. Res. Soc. Symp. Proc. 912, pp. 197-203, 2006.

[7]. www.capres.com.

[8]. S. Thorsteinsson, F. Wang, J-Y. Kim, D. H. Petersen, T. M. Hansen, D. Kjær, R. Lin, P. F. Nielsen, and O. Hansen, "Accurate microfour-point probe sheet resistance measurements on small samples," Rev. Sci. Instrum. 80, 053902(8pp), 2009.

[9]. D. H. Petersen, O. Hansen, R. Lin, and P. F. Nielsen, "Micro-four-point probe Hall effect measurement method," J. Appl. Phys. 104, 013710 (10pp), 2008.

[10]. T. Clarysse, J. Bogdanowicz, J. Goossens, A. Moussa, E. Rosseel, W. Vandervorst, D.H. Petersen, R. Lin, P.F. Nielsen, O. Hansen, G. Merklin, N.S. Bennett, and N.E.B. Cowern, "On the analysis of the activation mechanisms of sub-melt laser anneals," Mater. Sci. and Eng. B, 154, 24 (2008)

[11]. D. H. Petersen, O. Hansen, R. Lin, P. F. Nielsen, T. Clarysse, J. Goossens, E. Rosseel, and W. Vandervorst, "High precision micro-scale Hall effect characterization method using in-line micro four-point probes," in 16th IEEE International Conference on Advanced Thermal Processing of Semiconductor ( RTP' 2008), pp. 251-256, 2008.

[12]. F. Wang, D. H. Petersen, T. M. Hansen, P. Bøggild, and O. Hansen, "Sensitivity study of micro four-point probe measurements on small samples," J. Vac. Sci. Technol. B , in press. 